\title{
11
}

\section{Internal Benchmarking: Identifying Best Practices Within a Global Enterprise}

\section{Steve Crom 1}

BIBA (Bremen Institute of Industrial Science and Applied Work Science)

Hochschulring 20, D-28359 Bremen, Fax: + 494212185510

\section{INTRODUCTION}

As technology, customer expectations and competitive responses continue to change at an increasing rate, companies that learn the fastest have a competitive advantage. Internal benchmarking can create a network through which information and ideas are exchanged. It is an effective way of identifying and disseminating best practices from one part of a global enterprise to others, with the ultimate objective of improving business processes to deliver greater value to customers.

What follows is a discussion of internal benchmarking and the opportunities it offers as illustrated by the case of Globalco, a Fortune 100 consumer products company. The case is divided into the following sections:

- The company's situation and why internal benchmarking was appropriate.

- Gaining corporate sponsorship and selecting the right people for the benchmarking team.

- Building the team and defining its charter.

- Collecting the right data, qualitative and quantitative.

- Creating the final product.

- The results.

- Lessons learned about the process of internal benchmarking.

- Conclusions.

The discussion is based on the experience of the author working directly with this Fortune 100 company from August 1992 to May 1993 on a world-wide internal benchmarking study.

1 Thank you, Isabelle van Notten, for your editorial comments and contributions. 


\section{INTERNAL BENCHMARKING, THE OPPORTUNITIES IT OFFERS}

In many large multi-national companies there are pockets of excellence, aspects of an operating unit that represent leading edge practices from which there is a great deal to be learned. However, most organizations look externally and benchmark competitors and/or organizations in unrelated industries that have world class reputations in a particular area of interest, rather than learn from themselves. The advantages that internal benchmarking offers are as follows. It allows you to:

- $\quad$ probe for details, including what went wrong as well as right

- $\quad$ educate those doing the benchmarking to work in new ways

- demonstrate successes within your own company's culture and business environment

- establish communication channels and a network for highlighting and disseminating improvements and innovations throughout the organization

- focus external benchmarking activities once you know your own company's strengths and weaknesses

\section{GLOBALCO'S SITUATION AND WHY INTERNAL BENCHMARKING WAS APPROPRIATE}

Globalco prides itself in its entrepreneurialism and the autonomy given to country General Managers to run their operations as they see fit. The company established itself in over 60 countries by picking people who thrived on the challenge of creating a local business in difficult circumstances, often in developing countries with very little infrastructure and basic services i.e., a reliable supply of electricity. At this time, seventeen of the company's largest subsidiaries account for $80 \%$ of sales and profits, each developing, marketing, selling, producing, distributing similar products. As the businesses mature and face stiffer competition the question arises: How can a General Manager in one country learn about what is working in another country, take the idea, modify it to fit his/her circumstances and significantly improve the business?

David Bonasit, Vice President of Global Continuous Improvement, decided that benchmarking was a tool for identifying best practices throughout the company. He looked toward outside organizations: competitors and Best-In-Class non-competitors. A proposal for doing external benchmarking was developed by David and his team. When the proposal was reviewed with other executives, they commented:

"The culture in Globalco is such that successes developed internally are more credible than those that come from the outside. We do not have an accurate picture of what it is we do well." 
They therefore suggested that Bonisat visit certain operating units instead of looking outside. These Globalco operating units each exhibited excellence in several of the strategically important business processes. In response David Bonisat and his team decided to leverage the opportunity and benchmark eight key Globalco operating units or subsidiaries. Afterwards an external benchmarking study would be commissioned focused by the internal findings.

The options David considered for conducting an internal benchmark study were to hire an outside consulting firm or develop an internal team. First, as a former General Manager, David concluded that if a team of Globalco managers did the research themselves, packaged and communicated the results, these would be better accepted than if they came from an outsider. Second, members of the internal benchmarking team would become experts in benchmarking and be a valuable resource to others wanting to do benchmarking. Third, by selecting a cross functional team, David wanted to create a nucleus of influential managers who would help sell the application of leading edge practices throughout the company (in their respective functions), because they would discover themselves what differentiates successful from average practices. In addition to being cross-functional, David assembled a team that represented the company's divisions in Europe, Asia, North and South America to help spread the learning geographically.

David thought it would help to have a neutral person in the role of facilitator of team meetings and site visits. A consultant was selected based on his facilitation skills as well as experience in benchmarking and organizational development. The next big step was to get corporate support and the commitment to free up the right managers internally to participate in this seven month project.

\section{GAINING CORPORATE SPONSORSHIP AND SELECTING THE RIGHT PEOPLE FOR THE BENCHMARKING TEAM}

David sold the President of Globalco on the idea of an internal benchmarking team saying that the more involvement there is from the regions and functions at this stage the more commitment there will be to implement best practices globally. "Benchmarking is only as good as the operating improvements and results it leads too," David asserted. "While it takes longer, an internal team is in the best position to help translate the findings into concrete operating improvements." The proposal for doing internal benchmarking with a dedicated team of Globalco managers was approved.

The higher the caliber of the people on the team the more stock General Managers worldwide would put into the findings. David selected the members of the team based on who had reputations for being:

$\begin{array}{ll}\text { - } & \text { opinion leaders } \\ \text { - } & \text { functional experts } \\ \text { - } & \text { able to work in groups }\end{array}$

Each team member was expected to devote 25 to 30 days to the project. 
Three senior managers from David's staff with extensive experience in leading improvement efforts were apart of the team. Harold Pinter, the Continuous Improvement Manager responsible for South America was chosen as the project manager given his experience in data collection and quality audit programs. Before joining Globalco he learned about quality and Total Quality working in the automobile industry. Harold had exactly the right personality for the job of project manager. With a sense of humor, he kept the group organized, educating them along the way about benchmarking and the art of data gathering.

David and Harold picked a consultant, Scott Beckman, who was experienced in benchmarking but would leave the ownership of the project to Globalco, acting as a facilitator for the process and team meetings. Having an outside facilitator meant that David and Harold were free to participate in the team's working sessions while Scott kept the team focused and made sure that there was balanced participation. Each team member's role was clarified during a week long team building and planning event that launched the project.

\section{BUILDING THE TEAM AND DEFINING ITS CHARTER}

The more team members knew about the state-of-the-art in manufacturing and knew about each other, the more they could contribute to the project. Accordingly, Harold arranged the first team sessions to be held in Austin Texas, immediately following an international conference at which companies were presenting best practices in supply chain management. The first three days of the week the team attended presentations, bringing back insights about what they had heard. Most importantly the team members had time over dinner to get to know one another and learn how they wanted to contribute to the project.

Immediately following the conference the team spent two days off-site in a hotel room working on the following:

\section{Day 1 - Building The Team}

- The team's mission

- $\quad$ Each individual's learning style

- The team's learning style

- A strategy for working effectively together

- The role of each team member

\section{Day 2 - Defining The Team's Charter \& Task}

- The team's charter

- Categories of data to collect

- A protocol for gathering data

- The site visit schedule

- Pre-work requested of the host country before the visit

- Calendar of the project and team events 
As team leader David made it clear at the outset of day 1 that the team's mission was to document best practices and not to make recommendations during our benchmarking visits about what should be improved or how. The team wrestled with the question of what value they would offer the host country if they did not make improvement recommendations. "Too often," David said, "corporate task teams descend on a subsidiary, get a partial picture of what is going on, and leave the GM with a list of things to do as if he didn't already have enough to do." Instead, the benchmarking team would be on site to document the work processes and/or managerial practices that the subsidiary feels contributed the most to excellent customer service. Documenting best practices, how they were achieved and the principles involved turned out to be a service much appreciated and valued by the host country. It motivated them to continue the work they were doing with a renewed focus on the critical success factors.

At the end of the two day off-site meeting, the scope of the team's inquiry was defined as the total supply chain, from ordering raw material to delivering finished goods to the customer. Included in this definition was sales, marketing and product development activities. The team agreed that understanding excellent customer service practices meant identifying enabling factors such as leadership, organizational climate, training and development that contributed to creating and maintaining outstanding business processes and results.

\section{COLLECTING THE RIGHT DATA, QUALITATIVE AND QUANTITATIVE}

Practices that contributed to excellent customer service, on-time and complete deliveries in particular, was what the team set out to understand and document in a way that would be useful to others. The sites to visit were selected based on their reputation for excellent customer service, and historical on-time/complete delivery performance. Subsidiaries were selected from each of the company's geographic regions representing markets in which the company had dominant market shares as well as those in which it did not.

David called the General Managers of each subsidiary nominated, explained why he and the President had undertaken the project and enlisted their support in sharing successes throughout the company. Because of David 's reputation as a results oriented General Manager the subsidiaries agreed to host the team's visit.

The General Manager of each subsidiary received a pre-work packet from the Best Practices Team before they arrived on site. The packet contained:

- a description of the project, the team's charter and members

- advice on nominating successes or best practices to be documented

- a fact sheet to be filled out on each nominated best practice

- an agenda for the team's visit

- an explanation of how the team would go about collecting data once on site 
The prework package requested that the General Manager meet with his/her staff and identify the practices of which the subsidiary was most proud. They were asked to provide performance measures documenting improvement, plus a high level flow chart of the current process. The Best Practices Team reviewed the pre-work data before the visit, deciding which of the practices should be documented and by whom.

The site visits were scheduled to last three days. A typical visit started by meeting with the General Manager and his/her staff. In this initial meeting introductions were made, the team's purpose was reiterated as documenting the subsidiary's customer service successes. The General Manager made opening remarks about the subsidiary's performance and the context within which to place the subsidiary's results (e.g., social, political, economic, market and competitive conditions). Next, Scott facilitated a group discussion of the important historical developments that led to today's performance. In particular:

- What were things like before the current General Manager arrived?

- When was the current management team formed?

- Where there changes in organizational structure?

- What prompted the focus on customer service?

- How was support galvanized among employees to change?

- Were consultants involved? If so, how were they selected?

- How did the management team measure progress?

- What were the results over-time?

- What was the General Manager's role and the management team's role throughout?

- What would they do differently next time?

This opening conversation gave the Best Practices Team the same background information and allowed them to all see the General Manager and his/her staff in action together. In the final analysis the role of the General Manager as team leader and champion of customer service excellence surfaced as the single most important enabler of business process improvement.

At the close of the opening discussion the list of practices nominated by the subsidiary as their best were prioritized. Members of the management team formed small working groups with their functional counterparts on the Best Practices Team and another team member. The task of the working groups was to quantify the customer service benefit of specific practices, create a flow chart of the processes, show the magnitude of improvements made and document how the changes were made. Lessons learned and suggestions for how to get started were solicited during the interviews.

In the opening meeting and small work group meetings, improvement projects surfaced that merited documenting though they were not mentioned in the pre-work exercise. In fact, some of the better cases were not included in the pre-work. The team quickly learned that there was no substitute for face-to-face discussions, "walking the processes" and meeting with everyone involved including customers and suppliers. 
The data collection protocol used during the site visits was the following:

\section{Data Collection Protocol}

- Clearly define the business process, improvement goal, measures and implementation time frame.

- Identify the motivation for improvement. How was a sense of urgency to change created? Was there a competitive threat? Was the improvement goal focused on the customer?

- Capture the resources (people, expenses, outside consultants) that were involved.

- Flow chart the process as it was and it is in its improved form.

- List the people involved in the project, including the sponsor, the team leader, team members and supporting members paying special attention to their selection criteria, qualifications and roles.

- Document the improvement methodused and the steps involved.

- Write down the story of what happened and how it happened, in as much detail as possible. What was unique about this project? Capture any anecdotal stories about the team and the project to communicate to others how they worked together.

- Probe for data and results beyond the performance measures submitted in the pre-work. Be sure you have the latest data.

- Document the reasons for any backsliding or reversals in performance.

- Document the team's plans for continuing to improve the process.

- Explore what they would have done differently. What advice would he/she give others embarking on a similar process improvement effort?

The keys to capturing useful information were:

- Before conducting the first interviews generate a prototype of what a finished write-up should look like so everyone has the same end product in mind.

- Have a standard protocol that each member of the team follows to collect qualitative as well as quantitative information.

- Quantify performance and bottom-line benefits.

- Follow the flow of the business process as if you were a component of the process i.e., walk the process.

- Talk to those doing the work to really understand how it works, not how it is supposed to work. 
In daily debriefs, each member made a short presentation of what was documented and reported progress. Other team members were called on to explore a particular best practice that involved their functional expertise. Next steps were identified and the schedule adjusted accordingly.

The third day of the site visit, the team met to agree on which best practices and enablers would be written up. Scott listed them on flip chart to review with the management team. The agenda for the wrap-up session with the management team was:

- $\quad$ Review the list of best practices to be written up

- Review enablers of those best practices

- Ask how to package the findings in a way that would be most helpful to others

- Ask for feedback on the best practice team's approach and how it could have been improved

- Agree on a schedule for drafting the cases and getting feedback from the subsidiary before publication

- Summarize each team member's view of the best practices they documented

Validating the team's findings and correcting any misconceptions right away was the main purpose of the wrap-up. It was an opportunity to formally thank everyone involved. According to Harold, "in every visit the wrap-up was a smashing success because in every case the management teams had never really sat down and reviewed all the good things they had done over the past two or three years. It was a chance for their contributions to be recognized by a group of peers."

Before leaving the subsidiary, the team would document who was responsible for documenting each best practice and establish a documentation/review schedule. Upon returning to their home offices, each team member would prepare their reports and forward them to Harold Pinter according to the schedule. Some team members brought laptop computers to the site visited which allowed them to summarize their notes on the trip home. Harold would compile the draft reports and circulate them to the team and the subsidiaries. Both the team and the subsidiary personnel would review the cases and make corrections and additions as needed.

In summary, the documentation process was to:

1. Send pre-work to the host to identify best practices ahead of time.

2. Review the completed pre-work as a team before the visit.

3. Conduct an introductory meeting with senior management to set the tone, get to know the style of the management team and clarify the best practices to document. 
4. Meet in small working groups to collect as much detailed information as possible.

5. Debrief as a team daily to share impressions and adjust the data collection schedule.

6. Follow-up on site to fill as many data gaps as possible.

7. Conduct a wrap-up meeting on-site to validate the team's findings, thank the host and clarify next steps.

8. Review the draft cases with the team and host following the visit so everyone is comfortable that the information being published is accurate.

From Harold and David's perspective, value was added and knowledge gained in every step. At the same time, everyone was amazed at the amount of work, focus and persistence necessary to get a detailed, accurate and useful story.

\section{CREATING THE FINAL PRODUCT}

When asked how to best package the team's findings there was consensus that a book of write-ups by itself would not be very helpful. First, the challenge was to help people see the importance of customer service, to create a sense of urgency to change; second, to capture people's imagination about what was possible; third, to provide useful examples of how others have solved common problems; and last, to provide support in developing a coherent process improvement strategy and implementing it.

Compiling the final handbook was a big job. The corrected drafts from the subsidiaries, were given to an editor. In the process of polishing the text, the editor (a neutral party reading the cases without any particular functional expertise) prepared a list of items that needed clarifying. "This was a critical step in making the cases accessible to the widest possible audience within Globalco," remembers Harold. The editor's comments were forwarded to both the authors of the reports and the subsidiaries for their input. At this point, the cases were given to a desktop graphics person to format the final reports and integrate the graphics with the text. There were a total of over 80 cases and 500 pages of text.

The team developed a method so that operating unit management and improvement teams, the primary audience of this effort, could identify and prioritize the over 80 cases to select those most useful to them. The team developed a one page self-assessment form in which management or improvement teams would evaluate their current Customer Service status. The evaluation attributes were cross-referenced with the various cases. The assessment form was piloted, reviewed with operating unit management teams that were visited by the team, and revised accordingly. David Bonisat made his staff available to work with the operating units to facilitate the assessment and develop their action plans based on the best practice cases. 
Before the handbook was published, the Best Practice cases were reviewed with key stakeholders: Divisional Presidents and Vice-Presidents, and functional Vice-Presidents. The final approval to publish came from the functional Vice-Presidents of human resources, manufacturing, finance, technology and sales. After their final edits and recommendations the handbook went to press.

The plan is to add best practices to the handbook as they are discovered and/or developed, for it to be an archive that grows and is updated continuously.

\section{THE RESULTS}

The first operating units to benefit from this study, even before it was published, were those who participated in the study and those functions that provided key personnel to serve on the team. Ideas flowed freely between these constituents and many ideas were adapted and quickly instituted. There were many ideas of how Customer Service and Customer Service Improvement should be approached, "this study provided some key parameters to a unified Globalco approach," says Harold. Benchmarking (both internal and external) led to the establishment of a re-engineering effort in one of Globalco's key operating units.

The goal now is to develop a world class Customer Service methodology that is supported by a full time staff from several geographic regions and all key functions: just like the internal benchmarking team. "Several of the team members have been promoted and are now in position to really leverage what they learned," Harold reports. "All the team members are very proud to have served on the team and say that it was one of the best experiences of their careers. The members know that they can call on each other for support at any time and they do just that."

The Best Practices Handbook will be useful for the smaller and emerging operating units. Bonisat's department is small and, in reality, can and should only focus on the larger 17-20 operating units. "The book with the assessment form is a great reference for the rest of Globalco: 50 or so operating units," says David. "This handbook provides them with a unique resource. The feedback has been very positive."

\section{LESSONS LEARNED ABOUT THE PROCESS OF INTERNAL BENCHMARKING}

Internal benchmarking is ideal for global enterprises that have pockets of excellence but a tradition of autonomy rather than collaboration. It is best done with a team of the company's own managers trained in the best method of collecting and analyzing data. Through their direct involvement the organization learns how to learn, a strategically important process or capability in itself. Comments from team members reveal how much they learned:

"Get high level corporate sponsorship. It is essential in order to free up the caliber of resources needed to command people's respect and attention. The best people are always the busiest people, already assigned to high priority activities. It is only at the top that high priority activities can be reallocated so the right people have the time to devote to the project." 
"Build a group of people that know one another and how to work effectively together. It is just as important as knowing what data to collect and how. When doing benchmarking the message is in the method. If you are gathering information about excellent performance it should be done in a highly professional, well organized way."

"Skilled facilitation accelerates the team's development and economizes the time the team spends together. That person can be an outsider or someone from within the company as long as they view their role as guide rather than team leader."

"The team leader has to be a senior manager of the company with the authority and resources to convert the benchmarking findings into action."

"The staff work required to keep the team's activities organized, educate the team about the benchmarking (find and distribute articles) and edit the best practice write-ups is substantial. Harold devoted $40 \%$ or his time to this project for six months."

"Be flexible in the site visits and take your direction from your hosts."

"Expect your host to be cautious about the purpose of your visit until you have a chance to discuss the team and it's charter face-to-face."

"Sending pre-work to your hosts in advance as a courtesy but do not expect it to be self-explanatory since internal benchmarking is not yet widely practiced and understood."

"Everyone on the team should collect information about the qualitative aspects of the company that contribute most to its success, the enablers."

"Devote several hours to interviewing the General Manager one-onone since his/her philosophy, values and behavior is the most important enabler to outstanding corporate performance."

For those benchmarking for the first time internationally, be sensitive to culturally appropriate behavior. If team members are not experienced in traveling to a host company destination, give them material to read ahead of time so they avoid basic cultural faux pas. A special warning to Americans traveling to England, remember Mark Twain's admonition that "America and England are two countries divided by a common language." Don't assume that because you speak the same language (more or less) that the same behavior is appropriate in both countries. In probing for information, respect the protocol of asking senior managers for access to information before approaching middle level managers directly. 


\section{CONCLUSIONS}

If learning to improve processes is what separates the best companies from the average performers, it is in creating a learning network and environment that internal benchmarking has the most to offer. Think of the end-product of internal benchmarking as the building of a network and dynamic archive of knowledge rather than a final report. Build a team of the best resources available, managers who are most likely to move on to influential positions within the company. Assign a full-time team leader/coordinator. A key function that person fulfills is to communicate, communicate, communicate; it is an essential ingredient for the success of a global team. Take the team off-site to develop the team's ability to work together and clarify its own charter. Allow plenty of time early in the project for team building, planning and educating the team about state-of-the-art of benchmarking. Coordinating the first team events with a benchmarking conference is ideal.

Chose any consultant you work with carefully. Look for strong facilitation skills, benchmarking expertise and ability to fit into your corporate culture. His or her goal should be to transfer skills to the team as well as ensure the integrity of the process and data. Watch out for consultants who are more interesting in selling follow-on work than in successfully completing the benchmark study.

Involve the key stakeholders throughout the project. Get their visible endorsement of the project and commitment to use benchmarking to drive process improvement. Ask for their input on who should be including on the benchmark team. If senior executives are involved in the team selection process, they can help identify influential managers and will be more amenable to freeing up their time. This applies as well to selecting the sites to visit. Regular briefings and a final review of the practices documented with stakeholders helps keep the team focused on the company's top priorities.

Dedicate the resources necessary to create a world class product. Engage a professional editor and desktop publishing resource. To be a useful reference the final product must include contacts names (phone and fax numbers) for each best practice documented. A system should be created whereby a best practices archive can be kept, updated and accessed electronically by interested parties world-wide.

For internal benchmarking to be useful it has to be coupled with the resources to implement process improvement. While the two activities must be integrated, it is most effective for an internal benchmarking team to document best practices and not make improvement recommendations to their benchmark hosts. A self-assessment guide helps the users sort out the information that is most relevant. Above all, a practical process improvement strategy/plan must be in place that is supported by the company's leadership. 